\title{
A NUMERICAL STUDY OF THE INFLUENCE OF TEMPERATURE FLUCTUATIONS IN THE THERMAL RADIATION FIELD
}

\author{
E. D. dos Santos , \\ M. M. Galarça ${ }^{a}$, \\ A. C. Mossi ${ }^{a}$, \\ A. P. Petry ${ }^{\mathrm{a}}$, \\ and F. H. R. França ${ }^{a}$ \\ ${ }^{\text {a }}$ Universidade Federal do Rio Grande do Sul \\ Departamento de Engenharia Mecânica \\ Rua Sarmento Leite, 425 \\ CEP 90.050-170, Porto Alegre, Brasil \\ frfranca@mecanica.ufrgs.br

\section{NOMENCLATURE} \\ D diameter of the cylindrical chamber, $m$ \\ I total radiation intensity, $\mathrm{W} / \mathrm{m}^{2}$ \\ $\mathrm{I}_{\eta} \quad$ radiation intensity at a wavenumber $\eta, W / \mathrm{m}^{2} \mathrm{sr}$ \\ $\mathrm{h}$ height of the cylindrical chamber, $\mathrm{m}$ \\ $\mathrm{L}$ distance between two black walls, $\mathrm{m}$ \\ $\mathrm{q}_{\mathrm{R}} \quad$ radiative flux, $\mathrm{W} / \mathrm{m}^{2}$ \\ $\nabla \mathrm{q}_{R}$ divergence of radiative flux, $\mathrm{W} / \mathrm{m}^{3}$ \\ $\mathrm{T}$ temperature, $\mathrm{K}$ \\ $\mathrm{W}_{1} \quad$ weighting function \\ $r \quad$ radius of the cylindrical chamber, $m$ \\ $\mathrm{x}$ radiation path length, $\mathrm{m}$
}

\section{Greek symbols}

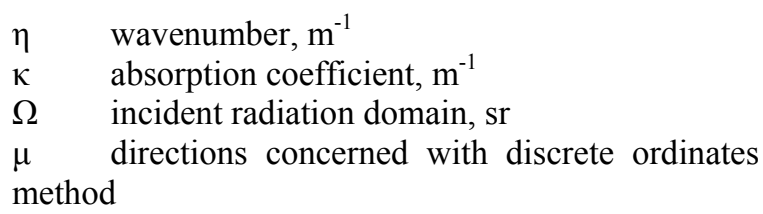

\section{Subscripts}

b blackbody

$\eta \quad$ radiant properties at a specific wavenumber

\section{Superscripts}

$+\quad$ positive direction of radiation intensity

- $\quad$ negative direction of radiation intensity

\section{INTRODUCTION}

Turbulent flows are present in many applications, such as aerodynamics, turbomachinery, thermal equipment and devices, combustion process and others. For non-isothermal flows it is also necessary to study the heat transfer mechanisms. Among them, the radiative heat transfer dominates the physical phenomena when high temperatures are present, i.e., it is important for the study of many engineering applications, for example: combustion, flames and rockets propulsion systems. According to Jones and Paul (2005) this mechanism has also become important for the development of thermal devices. For instance, the inaccurate prediction of temperature surfaces of a combustor can lead to an excessive amount of air entering in the combustion chamber, reducing the efficiency of the equipment as well as the average time between failures and increasing the pollutant emission.

Turbulent flows with convection heat transfer naturally generate fluctuations of scalar fields, temperature and species concentration. These fluctuations cause other ones in the absorption coefficient, which correlates with the fluctuations of the Planck function and the incident radiation intensity. These modifications in the thermal radiation field modify the energy sources that influence the fluid dynamic and thermal behavior of turbulent flows, i.e., changes in one field influence the behavior of the other one. These interactions are commonly named Turbulence-Radiation Interactions (TRI) (Li and Modest, 2003; Tessé et al., 2004; Modest, 2005; Coelho, 2007). However, the highly non-linear nature of these interactions can cause several difficulties for the numerical treatment. Moreover, turbulence and thermal radiation in participating medium are extremely complex phenomena, even when treated in an isolated form (Silveira Neto, 2002; Siegel and Howell, 2002; Lesieur et al., 2005). As a consequence, the traditional modeling of turbulent flows with thermal radiation heat transfer has generally ignored 
turbulence-radiation interactions, i.e., the divergence of radiative heat flux have been based on the average temperature and species concentration, which is in disagreement with encountered in experimental analyses (Li and Modest, 2002).

The studies on TRI originated in the 1960's. Nevertheless, the first numerical study was realized only in 1978 by Germano (1978), who had the sole interest to show the existence of turbulence-radiation interactions. Later, numerical studies on TRI consisted of solving the radiative transfer equation (RTE) directly using an experimental or imposed temperature field, this methodology is known as stochastic method (Kounalakis et al., 1988; Malalasekera et al., 2002; Coelho, 2004). Recently, the radiative transfer equation has been solved in a time averaged way, which consists of employing for the latter equation the same treatment used for time averaged mass, momentum and energy equations. This methodology is knowledge as Reynolds Averaged Navier-Stokes Equations (RANS) (Li and Modest, 2003; Tessé et al., 2004; Mazumder and Modest, 1999; Wang et al., 2008). However, the fluctuations of the correlations between the absorption coefficient and the Planck function and also of the absorption coefficient and incident radiation intensity in this method are not natural, being necessary to impose them by a probability density function (PDF). In the last years, Large-Eddy Simulations (LES) and Direct Numerical Simulation (DNS), which naturally generates the fluctuations of scalar fields, have been employed for the solution of conservation equations of mass, momentum and energy, as well as, for the radiative transfer equation. One difficult for the application of these methodologies is the enormous computational effort required. In this sense, they have been employed only in very simple problems, such as one or twodimensional simple domains, gray gases or ignoring the fluctuations in the calculation of radiative transfer equation. Thus, these approaches have contributed more to the understanding of the physical phenomena than to applications in real problems (Deshmukh et al., 2008; Roger et al., 2009; dos Santos et al., 2008).

The main goal of the present work is to evaluate the influence of temperature fluctuations in the thermal radiation fields, simulating the effect of turbulence-radiation interactions (TRI). To achieve this objective it is considered one-dimensional temperature profile with black walls filled with participating medium, which represents the behavior of a flame in a cylindrical chamber. Four temperature profiles were compared: an average and other three with $10 \%, 20 \%$ and $30 \%$ of turbulence intensity imposed over the averaged one. Some methodologies for the treatment of participating medium have been employed in the literature, such as: weighted-sum-ofgray-gases (WSGG) (Hottel and Sarofim, 1967; Smith et al., 1982), the spectral-line weighted-sumof-gray-gases (SLW) (Denison and Webb, 1993), the full-spectrum correlated-k distribution (Modest and Zhang, 2002) and the cumulative wavenumber (Solovjov and Webb, 2002), including studies by this research group (Mossi et al., 2008; Galarça et al., 2009). Although there are newest models which consider the complex spectral participating media behavior, it is employed in the present study the simplest approach for the treatment of nonisothermal, homogeneous, participating medium, i.e., the gray gas model. Two reasons explain this choice, the first one is related with the minimal computational effort required by the gray gas model; the second one concerns the intention to couple the code employed in the present work with another one developed by the present group (dos Santos, 2007; dos Santos et al., 2008; dos Santos et al., 2009) for the estimative of turbulent, transient, incompressible, two- and three-dimensional flows with forced and mixed convection heat transfer by means of LES, becoming possible the investigation of TurbulenceRadiation Interactions (TRI). The discrete ordinates method (DOM) is used to solve the radiative transport equation (RTE).

\section{MATHEMATICAL MODELING}

For the determination of the thermal radiation field it is necessary to solve the radiative transfer equation, which for a non-scattering medium is given by:

$$
\frac{d I_{\eta}}{d x}=-\kappa_{\eta} I_{\eta}+\kappa_{\eta} I_{b \eta}
$$

where $I_{\eta}$ is the spectral intensity $\left(\mathrm{W} / \mathrm{m}^{2} \mathrm{sr}\right)$ at wavenumber $\eta\left(\mathrm{m}^{-1}\right), \mathrm{I}_{\mathrm{b} \eta}$ is the blackbody intensity $\left(\mathrm{W} / \mathrm{m}^{2} \mathrm{sr}\right)$, and $\kappa_{\eta}$ is the spectral absorption coefficient $\left(\mathrm{m}^{-1}\right)$. The above equation can be solved a priori for every single value of $\kappa_{\eta}$, but it would lead to a large computational effort that would be prohibitive for most cases, with exception perhaps of onedimensional geometries.

Nevertheless, when the gas is considered gray, Eq. (1) becomes independent of the wavenumber $\eta$ and the radiative transfer equation could be rewritten by the following expression:

$$
\frac{\mathrm{dI}}{\mathrm{dx}}=-\kappa \mathrm{I}+\kappa \mathrm{I}_{\mathrm{b}}
$$

A noteworthy fact is that for a real gas the behavior of the absorption coefficient as function of wavelength is significantly different from the one estimated by the gray gas model. Thus, this simplification is used with the sole interest of understanding some characteristics of thermal radiation without considering the complications concerned with the spectral effects. One example is the study of the influence of the fluctuations of 
temperature field for the estimation of the divergence of the radiative flux, which is the scope of the present work.

With the purpose to couple the thermal radiation field and the other mechanisms of heat transfer (conduction and convection heat transfer) it is necessary to take into account one specific term concerned with the thermal radiation field to be inserted in the energy equation. Since the thermal radiation happens in an instantaneous time scale, naturally it can be inserted as a source term in the energy equation. This term is determined from the radiation intensity field and is known as divergence of the radiative flux $\left(\nabla \cdot \mathbf{q}_{\mathrm{R}}\right)$, which is given by:

$$
\nabla \cdot \mathbf{q}_{\mathrm{R}}=\int_{\eta=0}^{+\infty} \int_{4 \pi} \kappa_{\eta}\left(\mathrm{I}_{\eta \mathrm{b}}-\mathrm{I}_{\eta}\right) \mathrm{d} \Omega \mathrm{d} \eta
$$

For a gray gas, Eq. (3) can be simplified, since there is no dependence on the wavenumber. Then, the divergence of the radiative flux is rewritten as:

$$
\nabla \cdot \mathbf{q}_{\mathrm{R}}=\kappa\left(4 \pi \mathrm{I}_{\mathrm{b}}-\int_{4 \pi} \operatorname{Id} \Omega\right)
$$

\section{NUMERICAL MODELING}

As previously stated, the radiative transfer equation for the gray gas, Eq. (2), is solved here by the discrete ordinates method. Then, Eq. (2) is rewritten in the following form:

$$
\mu \frac{d I}{d x}=-\kappa I+\kappa I_{b}
$$

where $\mu$ represents the directions where the equations are solved.

Since the intensities can be found along each positive or negative cosine direction, Eq. (5) is shared in two equations, one for positive direction and other for negative direction, as can be seen:

$$
\begin{gathered}
\mu \frac{\mathrm{dI}^{+}}{\mathrm{dx}}=-\kappa \mathrm{I}^{+}+\kappa \mathrm{I}_{\mathrm{b}} \\
-\mu \frac{\mathrm{dI}^{-}}{\mathrm{dx}}=-\kappa \mathrm{I}^{-}+\kappa \mathrm{I}_{\mathrm{b}}
\end{gathered}
$$

Supposing that the thermal radiation transfer occurs between two black walls separated from a distance L, the boundary conditions of those surfaces are given by:

$$
\mathrm{I}^{+}(\mathrm{x}=0)=\mathrm{I}_{\mathrm{b}}(\mathrm{x}=0)
$$

$$
\mathrm{I}^{-}(\mathrm{x}=\mathrm{L})=\mathrm{I}_{\mathrm{b}}(\mathrm{x}=\mathrm{L})
$$

For the discretization of the terms concerned with the variation of intensity as function of radiation path, $d / d x$ for Eq. (6) - (7), it is employed the finite difference method (FDM).

Using the calculated gray gas intensities it is possible to obtain the net radiative flux, $\mathbf{q}_{\mathrm{R}}(\mathrm{x})$, and the divergence of the radiative flux, from the discrete ordinates method, through the following expressions:

$$
\begin{gathered}
\left.\mathbf{q}_{\mathrm{R}}(\mathrm{x})=2 \pi \sum_{1} \mu_{1} \mathrm{w}_{1} \mid \mathrm{I}^{+}-\mathrm{I}^{-}\right\rfloor \\
\left.\nabla \cdot \mathbf{q}_{\mathrm{R}}(\mathrm{x})=4 \pi \kappa \mathrm{I}_{\mathrm{b}}-2 \pi \kappa \sum_{1} \mathrm{w}_{1} \mid \mathrm{I}^{+}-\mathrm{I}^{-}\right\rfloor
\end{gathered}
$$

where $\mathrm{w}_{1}$ is the weighting function for each direction.

\section{DESCRIPTION OF THE PROBLEM}

It is analysed a flame into a cylindrical chamber with a radius of $r=10 \mathrm{~mm}$ and height of $\mathrm{h}=72 \mathrm{~mm}$. This flame was previously simulated by Barve and Ezekoye (2006) and the temperature topology predicted in that work is used here to generate the temperature profile. In the present work, it is evaluated a one-dimensional temperature profile for the transversal section located at the azimuthal coordinate $\mathrm{x}=12 \mathrm{~mm}$, transversal section AA. The sketch of the cylindrical chamber, as well as, the temperature topology of the simulation performed by Barve and Ezekoye (2006) can be observed in Fig. 1.

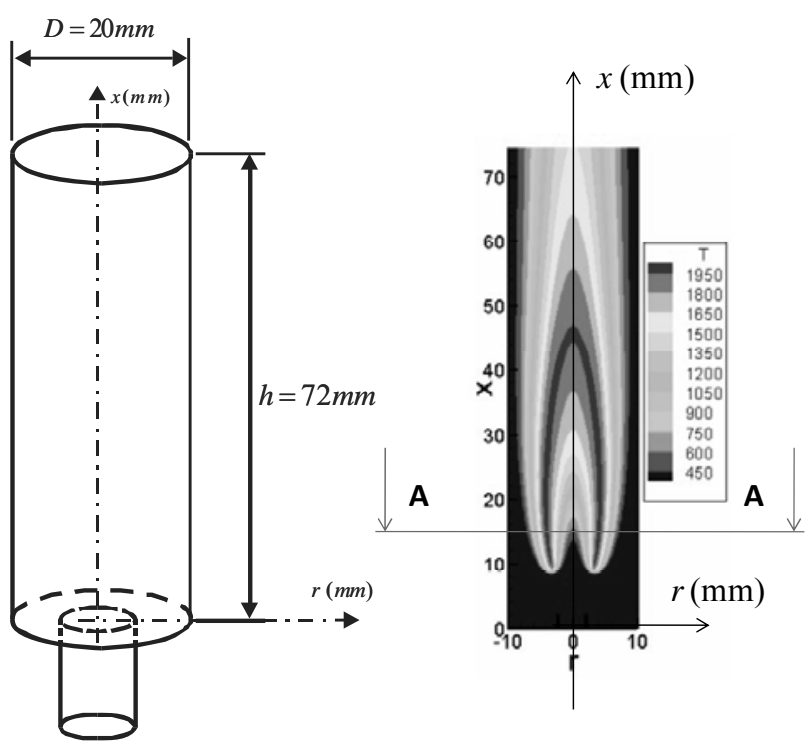

Figure 1. Sketch of the cylindrical chamber and temperature topology of the simulation performed by Barve and Ezekoye (2006). 
Figure 2 shows the description of the temperature profile obtained in the transversal section AA, at $x=12 \mathrm{~mm}$. Additionally, it is also presented in Fig. 2 the equation obtained for the temperature profile as function of the radius $r$.

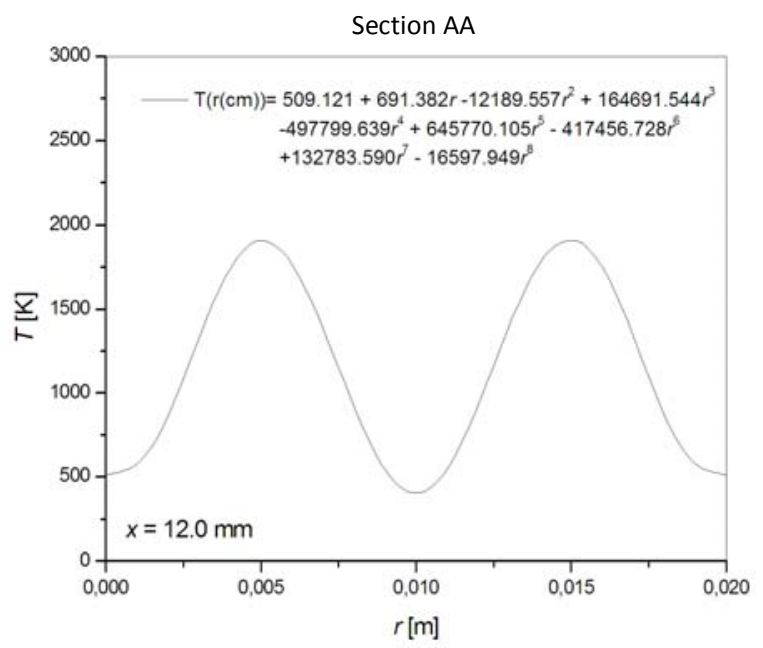

Figure 2. One-dimensional temperature profile as function of the radius $\mathrm{r}$ obtained at transversal section AA $-\mathrm{x}=12 \mathrm{~mm}$

\section{DESCRIPTION OF THE PROBLEM}

With the purpose to verify the estimative of the thermal radiation field from different methodologies of treatment of spectral properties, the SLW, CW, Gray Gas and LBL (Line-by-Line) methods are compared for the determination of the divergence of the radiative flux obtained from the average temperature profile, which is specified in the Fig. 2. These simulations show that there are various methodologies for the treatment of the spectral radiation properties, and these methodologies are also studied by this research group.

The LBL method led to benchmark solutions and is considered here as the reference. The participant medium is a mixture of $10 \%$ of $\mathrm{CO}_{2}$ and an inert gas (air or nitrogen, for instance). The SLW, $\mathrm{CW}$ and LBL methods, which are based in the gases spectrum, were calculated with the HITRAN04 database (Rothman et al., 2005). For the SLW and $\mathrm{CW}$ methods it was considered 20 gray gases logarithmically spaced between $1 \times 10^{-25} \mathrm{~cm}^{2} /$ molec. and $1 \times 10^{-17} \mathrm{~cm}^{2} /$ molec. Concerning the discrete ordinates method, 20 directions were used for all simulated cases. The independent grid was obtained with 200 volumes along of the radius of the chamber. For the gray gas model the absorption coefficient used was optimized through the analysis of the spectrum at the averaged temperature of the participating medium. The absorption coefficient of $0.03 \mathrm{~m}^{-1}$ led to the best results with gray gas model and it was used in the present simulations.
Figure 3 shows the divergence of the radiative flux obtained with LBL, CW with reference temperature of $402 \mathrm{~K}$, CW with reference temperature of $1912 \mathrm{~K}$, SLW and Gray Gas. It was observed that, the predictions of $\mathrm{CW}$ model had the best agreement with the ones predicted with LBL model, especially when the reference temperature is $1912 \mathrm{~K}$. The SLW model captured the tendency of the curve. However, it overestimates the divergence of the radiative flux for higher temperature gradients, being surprisingly worse than the estimative of the gray gas model in this region. The Gray Gas model, similarly to SLW model, just captured some tendency of the divergence of the radiative flux. Nevertheless, it presented the worst results for the surface regions.

Despite the fact that $\mathrm{CW}$ model led to results significantly better than the gray gas model, the latter was employed in the present work due to the need to minimize CPU time, since in the future the code to determine the thermal radiation field will be coupled with a developed code to simulate transient, threedimensional and turbulent flows by means of large eddy simulations (LES), which requires enormous processing time. Besides that, for three-dimensional domains the time processing of the thermal radiation code also increases significantly, reinforcing the need to employ the simplest methodology for the treatment of thermal radiation properties, at least for the first studies about TRI by means of LES. Thus, if the turbulence-radiation interactions present suitable behavior for a gray gas model, it is reasonable to accept that for the more detailed models, for instance, CW modeling, better predictions are achieved.

The divergence of the radiative flux is calculated from the averaged temperature profile, shown in Fig. 2 and Fig. 4, with the objective to estimate the influence of the average temperature field over the thermal radiation field. To estimate the influence of the fluctuations over the thermal radiation field, which simulates the influence of the turbulent flows, it was analyzed three temperature profiles with fluctuations imposed over the averaged temperature profiles, representing profiles with $10 \%$, $20 \%$ and $30 \%$ of turbulence intensity, as can be seen in Fig. 4. This procedure is similar to the ones employed by stochastic method, where the fluctuations are imposed over the temperature averaged profiles. With the purpose to avoid the increase of the mean of temperature profile by the insertion of the fluctuations, which could affect the mean of the divergence of radiative flux, the temperature profiles are constructed in such way that the mean of the average profile and the profiles with fluctuations are the same. 


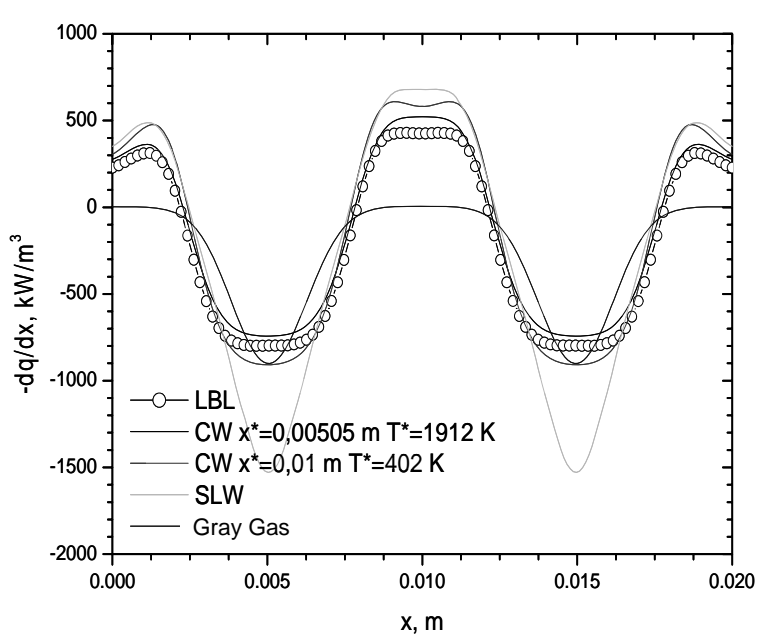

Figure 3. Comparison of the divergence of radiative flux obtained with LBL, CW, SLW and Gray Gas models from the averaged profile.

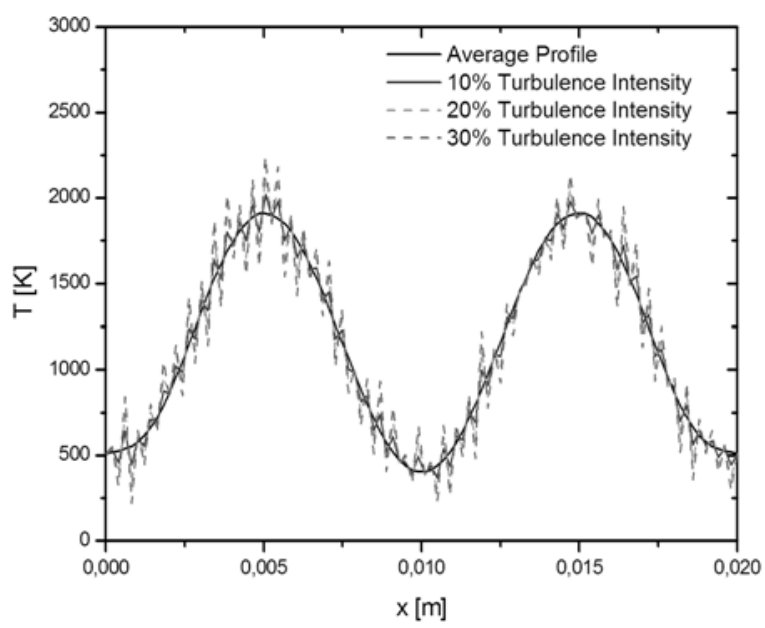

Figure 4. Temperature profiles: average, 10\%, 20\% and $30 \%$ of turbulence intensity

Figure 5 presents the results of the divergence of radiative flux predicted from the average temperature profile and other ones with fluctuations, simulating the effect of turbulence. It is observed a significant increase of the divergence of the radiative flux with the increase of the turbulence intensity, especially for higher temperatures in the domain. For lower temperatures, the divergence of the radiative flux tended to null values and the influence of the temperature fluctuations for the prediction of the radiative properties was neglected. This happens due to the emitted intensity for the temperature peaks to be more influencing than the valleys, since the emitted intensity is a forth power function of temperature.

Table 1 presents the mean of the divergence of the radiative flux estimated from the average temperature profile and the other ones with $10 \%$, $20 \%$ and $30 \%$ of turbulence intensity. It was verified that the mean divergence of the radiative flux increased significantly with the increase of the turbulence intensity of temperature profile, the divergence of the radiative flux predicted from a temperature profile with $30 \%$ of turbulence intensity was approximately $20 \%$ higher than the one obtained from the average temperature profile, corroborating the conclusions achieved from Fig. 5. The results obtained here agreed with the numerical and experimental predictions of Kounalakis et al. (1988), which stated that the mean radiation levels are higher when predicted from instantaneous scalar fields than when estimated from average scalar fields.

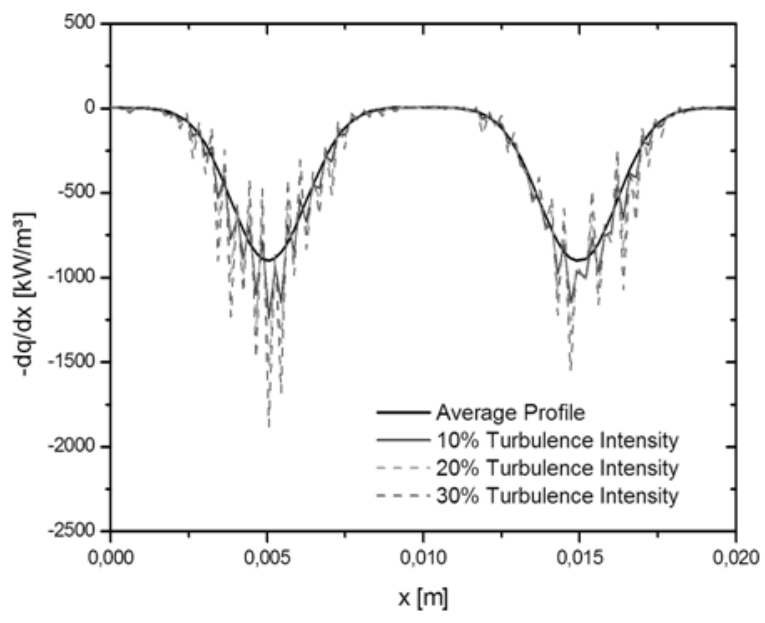

Figure 5. Divergence of the radiative flux for the temperature profiles: average, $10 \%, 20 \%$ and $30 \%$ of turbulence intensity

Table 1. Mean divergence of the radiative flux obtained from the average and instantaneous temperature profile

\begin{tabular}{ccc}
\hline $\begin{array}{c}\text { Temperature } \\
\text { profile }\end{array}$ & $\begin{array}{c}\overline{d \mathbf{q} / d r} \\
\left(\mathrm{~kW} / \mathrm{m}^{3}\right)\end{array}$ & $\begin{array}{c}\text { Deviation } \\
\mathbf{( \% ) *}\end{array}$ \\
\hline Average & 266.18 & ------------- \\
\hline $\begin{array}{c}10 \% \text { Turbulence } \\
\text { Intensity }\end{array}$ & 294.64 & $10.69 \%$ \\
\hline $\begin{array}{c}\text { 20\% Turbulence } \\
\text { Intensity }\end{array}$ & 303.74 & $14.11 \%$ \\
\hline $\begin{array}{c}30 \% \text { Turbulence } \\
\text { Intensity }\end{array}$ & 319.80 & $20.14 \%$ \\
\hline * Deviation $(\%)=\left[\left(d \mathbf{q} /\left.d r\right|_{\text {flut. }}-d \mathbf{q} /\left.d r\right|_{\text {mean }}\right) / d \mathbf{q} /\left.d r\right|_{\text {mean }}\right] \times 100$
\end{tabular}

\section{CONCLUSIONS}

In the present work, it was performed a numerical study on the influence of the fluctuations of temperature fields over the thermal radiation field, simulating the turbulence-radiation interactions. To evaluate the behaviour of the divergence of radiative heat flux for a flame in a cylindrical cavity, four temperature profiles were imposed: an average temperature profile and other three with $10 \%, 20 \%$ and $30 \%$ of turbulence intensity. The radiative 
transfer equation was solved with the discrete ordinates method (DOM) and the participant medium was treated as gray.

Additionally, various advanced gas models (SLW, CW and gray gas) were compared to the benchmark model (LBL). Although the results of the divergence of the radiative heat flux were better predicted by $\mathrm{CW}$ models than by gray gas, in the first applications of turbulence-radiation interactions (TRI), it will be employed the latter one to minimize the time processing.

It was observed in the simulations of the turbulence-radiation interactions a significative increase of the local divergence of the radiative flux with the increase of the turbulence intensity, especially for higher temperatures in the domain. It was also verified that the mean divergence of the radiative flux increase significantly with the increase of the fluctuations on the temperature profile, for a profile with $30 \%$ of turbulence intensity the difference of the mean divergence of the radiative flux in comparison to the one for an average temperature profile was approximately $20 \%$. The results obtained here agreed with the numerical and experimental predictions of Kounalakis et al. (1988).

\section{ACKNOWLEDGEMENTS}

The first author thanks CAPES by his doctorate scholarship, the second and third author thank CNPQ by their doctorate scholarship, F. H. R. França thanks CNPQ for research grant 304535/2007-9. All authors thank CAPES (Brazil) for the support under the program CAPES/UT-AUSTIN, No. 028/08.

\section{REFERENCES}

Barve, V. V., Ezekoye, O. A., 2006, Computations of Strongly Forced Laminar ColdFlow Jet and Methane-Air Diffusion Flames, MSc. Thesis, Texas University, Texas.

Coelho, P. J., 2004, Detailed Numerical Simulation of Radiative Transfer in a Non-luminous Turbulent Jet Diffusion Flame, Combustion Flame, Vol. 136, pp. 481-492.

Coelho, P. J., 2007, Numerical Simulation of the Interaction between Turbulence and Radiation in Reactive Flows, Progress in Energy and Combustion Science, Vol. 33, pp. 311-383.

Denison, M. K., and Webb, B. W., 1993, A Spectral Line Based Weighted-Sum-of-Gray-Gases Model for Arbitrary RTE Solvers, Journal of Heat Transfer, Vol. 115, pp. 1004-1012.

Deshmukh, K. V., Modest, M. F., and Haworth, D. C., 2008, Direct Numerical Simulation of Turbulence-Radiation Interactions in a Statistically One-Dimensional Non-Premixed System, Journal of Quantitative Spectroscopy \& Radiative Transfer, Vol. 109, pp. 2391-2400.
Dos Santos, E. D., 2007, Analysis of Nonisothermal, Incompressible Flows, Using Large Eddy Simulations and Finite Element Method, MSc. Thesis, Universidade Federal do Rio Grande do Sul, Porto Alegre, Brasil. (in Portuguese)

Dos Santos, E. D., Petry, A. P., and Rocha, L. A. O., 2008, Numerical Analysis of Non-Isothermal Lid-Driven Cavity Flows Using Large Eddy Simulation, in: Proceedings of the $12^{\text {th }}$ Brazilian Congress of Thermal Engineering and Sciences, Belo Horizonte.

Dos Santos, E. D., Piccoli, G. L., França, F. H. R., and Petry, A. P., 2009, Numerical Analysis of Transient Convection Heat Transfer Employing Two Different Temporal Schemes: Explicit Iterative of Taylor-Galerkin and Two Step Explicit, in: Proceedings of $20^{\text {th }}$ International Congress of Mechanical Engineering, Gramado.

Dos Santos, R. G., Lecanu, S., Ducruix, S., Gicquel, O., Iacona, E., and Veynante, D., 2008, Coupled Large Eddy Simulations of Turbulent Combustion and Radiative Heat Transfer, Combustion and Flame, Vol. 152, pp. 387-400.

Galarça, M. M., Mossi, A. C., Vielmo, H. A., França, F. H. R., and Ezekoye, O. A., 2009, Application of the Cumulative Wavenumber Model for a Non-Isothermal Homogeneous Medium Filled with $\mathrm{CO}_{2}$ or $\mathrm{H}_{2} \mathrm{O}$, in: Proceedings of $20^{\text {th }}$ International Congress of Mechanical Engineering, Gramado.

Germano, M., 1978, Turbulent Fluctuations Coupled with the Radiation Field, AIAA Paper, Vol. 78, pp. 780-840.

Hottel, H. C., and Sarofim, A. F., 1967, Radiative Transfer, McGraw-Hill Book Company.

Jones, W. P., and Paul, M. C., 2005, Combination of DOM with LES in a Gas Turbine Combustor, International Journal of Engineering Science, Vol. 43, pp. 379-397.

Kounalakis, M. E., Gore, J. P., and Faeth, G. M., 1988, Turbulence/Radiation Interactions in Nonpremixed Hydrogen/Air Flames, in: $22^{\text {nd }}$ Symposium (International) on Combustion, Pittsburgh, pp. 1281-1290.

Lesieur, M., Métais, O., and Comte, P., 2005, Large-Eddy Simulations of Turbulence, Cambridge, New York.

Li, G., and Modest, M. F., 2002, Application of Composition PDF Methods in the Investigation of Turbulence-Radiation Interactions, Journal of Quantitative Spectroscopy \& Radiative Transfer, Vol. 73, pp. 461-472.

Li, G., and Modest, M. F., 2003, Importance of Turbulence-Radiation Interactions in Turbulent Diffusion Flames, Journal of Heat Transfer, Vol. 125, pp. 831-838.

Malalasekera, W., Versteeg, H., Henson, J. C., and Jones, J. C., 2002, Calculation of Radiative Heat Transfer in Combustion Systems, International 
Journal of Energy Combustion Technology, Vol. 3, pp. 113-143.

Mazumder, S., and Modest, M. F., 1999, Turbulence-Radiation Interactions in Nonreactive Flow of Combustion Gases, Journal of Heat Transfer, Vol. 121, pp. 726-729.

Modest, M. F., 2005, Multiscale modeling of turbulence radiation and combustion interactions in turbulent flames, International Journal of Multiscale Computations Engineering, Vol. 3, pp. 85-106.

Modest, M. F., and Zhang, H., 2002, The FullSpectrum Correlated-k Distribution for Thermal Radiation from Molecular Gas-Particulates Mixtures, Journal of Heat Transfer, Vol. 124, pp. 30-38.

Mossi, A. C., Galarça, M. M., and França, F. H. R., 2008, Modeling of the Radiative Heat Transfer by CW Model in Gaseous Media, in: Proceedings of the $12^{\text {th }}$ Brazilian Congress of Thermal Engineering and Sciences, Belo Horizonte.

Roger, M., Da Silva, C. B., and Coelho, P. J., 2009, Analysis of the Turbulence-Radiation Interactions for Large Eddy Simulations of Turbulent Flows, International Journal of Heat and Mass Transfer, Vol. 52, pp. 2243-2254.

Rothman, L. S., Jacquemart, D., Barbe, A., Benner, D. C., Birk, M., Brown, L. R., Carleer, M. R., Chackerian Jr., C., Chance, K., Coudert, L. H., Dana, V., Devi, V. M., Flaud, J. M., Gamache, R. R., Goldman, A., Hartmann, J. M., Jucks, K. W., Maki, A. G., Mandin, J. Y., and Massie, S. T., 2004, The HITRAN 2004 Molecular Spectroscopic Database, Journal of Quantitative Spectroscopy \& Radiative Transfer, Vol. 96, pp. 139-204.

Siegel, R., and Howell, J. R., 2002, Thermal Radiation Heat Transfer, Taylor \& Francis, New York.

Silveira Neto, A., 2002, Turbulência, Vol. I, Editors: Silva Freire, A. P., Menut, P. P. M., Jian, S., ABCM, Rio de Janeiro.

Smith, T. F., Shen, Z. F., and Friedman, J. N., 1982, Evaluation of Coefficients for the Weighted Sum of Gray Gases Model, Journal of Heat Transfer, Vol. 104, pp. 602-608.

Solovjov, V. P., and Webb, B. W., 2002, A Local Spectrum Correlated Model for Radiative Transfer in Non-uniform Gas Media, Vol. 73, pp. 361-373.

Tessé, L., Dupoirieux, F., and Taine, J., 2004, Monte Carlo Modeling of Radiative Transfer in a Turbulent Sooty Flame, International Journal of Heat and Mass Transfer, Vol. 47, pp. 555-572.

Wang, A., Modest, M. F., and Haworth, D. C., 2008, Monte Carlo Simulation of Radiative Heat Transfer and Turbulence Interactions in Methane/Air Jet Flames, Journal of Quantitative Spectroscopy \& Radiative Transfer, Vol. 109, pp. 269-279. 